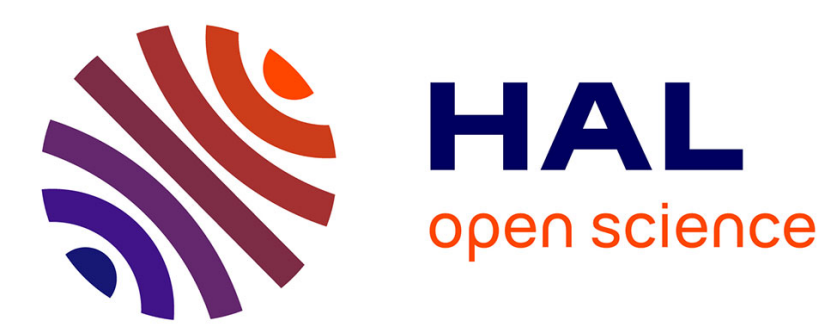

\title{
Les maraîchers cévenols du réseau Nature et Progrès, des agriculteurs invisibles?
}

\author{
Aurélie Javelle, Hélène Tallon
}

\section{To cite this version:}

Aurélie Javelle, Hélène Tallon. Les maraîchers cévenols du réseau Nature et Progrès, des agriculteurs invisibles ?. Pour: enquêtes et témoignages, 2017, 231 (2016/3), pp.9-14. 10.3917/pour.231.0009 . hal-02617579

\section{HAL Id: hal-02617579 \\ https://hal.inrae.fr/hal-02617579}

Submitted on 25 May 2020

HAL is a multi-disciplinary open access archive for the deposit and dissemination of scientific research documents, whether they are published or not. The documents may come from teaching and research institutions in France or abroad, or from public or private research centers.
L'archive ouverte pluridisciplinaire HAL, est destinée au dépôt et à la diffusion de documents scientifiques de niveau recherche, publiés ou non, émanant des établissements d'enseignement et de recherche français ou étrangers, des laboratoires publics ou privés. 


\section{LES MARAÎCHERS CÉVENOLS DU RÉSEAU NATURE ET PROGRÈS, DES AGRICULTEURS INVISIBLES ?}

Aurélie Javelle, Hélène Tallon

GREP | « Pour »

2016/3 N²31 | pages 9 à 14

ISSN 0245-9442

Article disponible en ligne à l'adresse :

https://www.cairn.info/revue-pour-2016-3-page-9.htm

\section{Pour citer cet article :}

Aurélie Javelle, Hélène Tallon « Les maraîchers cévenols du réseau Nature et Progrès, des agriculteurs invisibles ? », Pour 2016/3 ( $\left.\mathrm{N}^{\circ} 231\right)$, p. 9-14. DOI 10.3917/pour.231.0009

Distribution électronique Cairn.info pour GREP.

(c) GREP. Tous droits réservés pour tous pays.

La reproduction ou représentation de cet article, notamment par photocopie, n'est autorisée que dans les limites des conditions générales d'utilisation du site ou, le cas échéant, des conditions générales de la licence souscrite par votre établissement. Toute autre reproduction ou représentation, en tout ou partie, sous quelque forme et de quelque manière que ce soit, est interdite sauf accord préalable et écrit de l'éditeur, en dehors des cas prévus par la législation en vigueur en France. Il est précisé que son stockage dans une base de données est également interdit. 


\section{analyses et actualités}

\section{Agriculture}

\section{Les maraîchers cévenols du réseau Nature et Progrès, des agriculteurs invisibles?}

\section{Problématique}

La question de la coexistence des modèles agricoles est à l'ordre du jour. Parmi ceux-ci, l'agroécologie, qui devient une référence dans les politiques publiques. En effet, le Ministère en charge de l'Agriculture invite les acteurs du monde agricole à évoluer vers des pratiques agro-écologiques, définies comme triplement performantes: économiquement, socialement et écologiquement (MAAF 2013). En cela, il semblerait que la volonté politique institutionnelle rejoigne les démarches proposées par les agriculteurs du réseau Nature et Progrès, " une fédération de consommateurs et de professionnels engagés depuis 1964 dans l'agroécologie ${ }^{1} \gg$.

Or, aux dires de certains agriculteurs $N \& P$ cévenols, leurs systèmes de production agro-écologiques seraient peu reconnus, voire même contestés et discrédités par certains acteurs ${ }^{2}$. Cette remarque, entendue à une échelle locale, a fait écho en nous à la polémique nationale autour de la bio, suscitée par une étude de l'INRA sur les agricultures à haute performance $(2013)^{3}$, et qui illustre les tensions autour de la reconnaissance de l'agriculture biologique, en termes de productivité et de compétitivité. Ces réactions renvoient aux débats portant sur l'évaluation des systèmes de production dits non conventionnels, atypiques, alternatifs, etc., mais aussi à la marginalisation des professionnels qui ne répondent pas aux critères technico-économiques dominants et définis a priori par les politiques agricoles. Si bien que nous nous posons la question, dans cet article, au travers d'un travail d'enquête de terrain, de l'invisibilisation des agriculteurs rencontrés, et par conséquence de leur place dans les politiques publiques.

\section{Terrain}

Notre étude porte sur les pratiques agroécologiques d'agriculteurs affiliés au réseau Nature et Progrès, situés dans les vallées cévenoles du sud Lozère. Un premier travail d'enquête sous forme d'observation participante a été mené entre 2012 et 2013 auprès des 10 maraîchers N\&P du territoire (parmi les 32 producteurs portant la mention N\&P), sur le thème de la gestion de la fertilité des sols ${ }^{4}$. Ce travail a été complété par deux stages de M2, 
en agronomie (Catalogna, 2014) et en ethnologie (De la Granville, 2016), basés sur des enquêtes qualitatives. Les maraîchers enquêtés ont entre 30 et 60 ans. Aucun n'est originaire de la région et n'a de formation initiale agricole. Ils se sont installés par vagues successives depuis les années 1970 sur d'anciennes fermes isolées à l'abandon, traditionnellement aménagées en terrasses pour répondre aux contraintes des terrains pentus de ces vallées étroites dont l'altitude varie de 400 à $650 \mathrm{~m}$. La surface moyenne d'une terrasse est de quelques centaines de mètres carrés. La propriété peut comporter fréquemment jusqu'à 40 hectares, mais seuls 1 ou 2 hectares sont exploités en maraîchage. Les productions (produits transformés, légumes, châtaignes, petits fruits, miel, plantes aromatiques ou médicinales...) sont vendues sur les marchés locaux ou à des coopératives, voire directement à la ferme, par exemple aux touristes. La faible productivité agricole, les risques liés à l'usage de variétés anciennes et aux expérimentations, la faiblesse des revenus dégagés de l'activité sont compensés par une forte pluriactivité, installée dans le temps.

Toutes ces personnes racontent qu'elles sont arrivées sur ces espaces par hasard, au fil des rencontres ou des voyages, et s'y être installées par désir de vivre selon des principes de respect de l'environnement et de valeurs sociales, l'agriculture étant le moyen de concrétiser cet engagement. Elles s'affirment de manière radicale contre un modèle «industriel, productiviste et réducteur de la diversité du vivant », auquel elles opposent des pratiques agroécologiques privilégiant un rapport holiste à la terre et aux éléments de nature. Pour cela, ces agriculteurs conçoivent et expérimentent des systèmes techniques valorisant les processus écologiques, la biodiversité, et surtout les savoirs et savoir-faire porteurs de ruptures ontologiques: rapport à l'incertitude, à l'ignorance, à l'intuition, aux connaissances sensibles, relation partenariale ou de don contre don avec les objets de nature. Les pratiques observées correspondent ainsi au principe d'écologisation de l'agriculture, posant la nécessité de penser la complexité (Girard, 2014) et accepter une action positive des éléments de nature (Barbier et Goulet, 2013). Un maraîcher installé depuis plus de 20 ans déclare: «Quand je demande quelque chose à la terre, il faut que je le rende, il faut que je m'en occupe, tu vois? Sinon la nature n'a pas besoin de toi, elle peut faire ce qu'elle veut. Mais à partir du moment où tu interfères, il faut quand même la respecter. ». D'un point de vue agronomique ces pratiques se traduisent par une gestion micro parcellaire des terrains, dans laquelle le geste «nécessaire » est substitué en partie au geste « rentable » pour assurer l'équilibre de la relation don/contre don sous entendue dans leur système. Pour autant les pratiques sont variées et les innovations agronomiques nombreuses : utilisation de buttes, mulchs plus ou moins complexes, travail minimal du sol, mélanges d'espèces etc. (Catalogna, 2014).

\section{Analyse}

Ces systèmes de production sont peu reconnus, malgré le travail d'appui mené par le réseau N\&P pour 
contribuer à leur diffusion. Le maraîchage réalisé sur des terrasses de faibles surfaces est considéré comme une pratique marginale. En effet, les productions en terrasse portent une connotation négative due à la pénibilité du travail et à la difficulté à le mécaniser, alors même que la mécanisation est depuis 50 ans symbole de progrès en agriculture. D'autre part, ce système de production agronomique particulier reste techniquement anecdotique en France. Il est ainsi invisible dans les statistiques agricoles nationales, mais aussi dans les données plus locales du Parc National des Cévennes ou de l'organisme de gestion du site «Bien Unesco », la zone étant inscrite sur la liste du patrimoine mondial de l'Unesco au titre de paysage culturel de l'agro-pastoralisme. A lire le document de gestion du site, les pratiques agricoles étudiées ici semblent quasiment absentes, le plan de gestion soulignant par exemple «la nécessité de réfléchir à l'avenir des terrasses aujourd'hui de moins en moins utilisées à l'échelle du territoire » Entente Départementale des Causses et Cévennes, 2014, p. 14). Plus largement, les producteurs N\&P sont peu mentionnés dans la recherche agronomique française. Seules un peu plus d'une centaine d'occurrences ressortent des diverses bases de données bibliographiques ${ }^{5}$, beaucoup d'entre elles étant liées à des questions sociopolitiques et non pas agronomiques. Pourtant, à y regarder de près, les innovations de ces agriculteurs N\&P, dont certaines peuvent apparaître comme des innovations de rupture, ne sont pas si loin des innovations conventionnelles du secteur : développement de l'agriculture biologique, non labour, etc., et les hybridations sont nombreuses: non travail du sol sur une parcelle mais travail moto-mécanisé sur une autre, mobilisation de savoirs empiriques et d'indicateurs sensoriels (vue, toucher, odorat) couplés aux savoirs «scientifiques » classiques (mesure du pH par exemple), etc.

A cette invisibilité «académique », s'ajoutent une invisibilité géographique et une invisibilité professionnelle. L'isolement géographique de ces maraîchers est manifeste. Ils vivent sur des communes très faiblement peuplées (de 7,5 à 17 hab./ $\mathrm{km}^{2}$ ), et leurs fermes, situées dans un territoire isolé et accidenté, sont difficilement accessibles, les «néo » en quête de foncier investissant les terrains libres, souvent les plus éloignés et les plus pauvres. Les conditions pédoclimatiques y sont peu favorables au maraîchage. A tel point qu'un couple se souvient des conseils de la Chambre d'Agriculture à leur arrivée en Lozère il y a une vingtaine d'années: « il faut pas s'installer, on peut pas vivre en Cévennes. Il faut pas venir! » Bien qu'accentuant leurs difficultés à produire, cette situation reste cohérente avec leur choix de vie qui résulte en grande partie d'une stratégie d'échappement (vivre là pour « être tranquille »), mais elle contribue à les mettre à l'écart. D'autant plus que la zone étudiée est identifiée localement comme une zone de "post-soixantehuitards militants altermondialistes », et que l'adhésion au label N\&P accentue la marginalité de ces agriculteurs en les différenciant idéologiquement et techniquement du monde de la bio. Pourtant, là encore, ces producteurs non seulement ne sont pas coupés de leur territoire mais participent 
activement à sa revitalisation. Ils défendent sa diversité culturelle, écologique et territoriale, et ancrent toutes leurs productions dans le territoire, les actes d'achat et de vente étant raisonnés selon des critères socioenvironnementaux, privilégiant autant que possible les circuits courts pour ne citer qu'un exemple.

Côté institutions, ces producteurs ne se sentent pas appuyés. L'usage des préfixes et suffixes «bio», «éco » et les termes «biologiques» et «écologiques» sont interdits pour les producteurs N\&P qui ne sont pas labellisés dans le cadre du Règlement Bio Européen ${ }^{6}$ et ne sont pas certifiés par un organisme tiers ${ }^{7}$. La situation est d'autant plus paradoxale que la fédération N\&P a été l'un des fondateurs du mouvement pour l'agriculture biologique, et a grandement contribué à la reconnaissance officielle de cette agriculture $^{8}$. Sans compter que le système de certification utilisé par N\&P lle Système Participatif de Garantiel est reconnu par l'IFOAM Monde 9 mais pas par le règlement européen. La situation semble traduire l'opposition entre les partisans d'un système majoritaire de certification et ceux d'un procédé d'assurance-qualité qui, selon eux, dépasse les limites d'une labellisation bio passant par un organisme tiers ${ }^{10}$.

Sur un autre plan, le choix de ces maraîchers de s'installer avec peu de capitaux leur permet de ne pas demander de DJA ${ }^{11}$ et de ne pas rentrer dans un système d'évaluation normative de la viabilité économique de leur installation qui les pousserait à une intensification du travail contradictoire avec leur choix de vie, comme le fait remarquer cette agricultrice qui a reçu une DJA et semble le regretter: " $A$ l'époque il fallait atteindre un certain revenu après les premiers 3 ans, pour toucher la 2ème moitié des sous qu'ils te filent. On a bossé comme des idiots [ ] A un certain moment, au niveau des revenus, on était largement au-dessus, on s'est dit "Qu'est-ce qu'on est en train de faire?» On n'avait pas le temps de faire le potager pour nous! On achetait des légumes! On n'était que en train de transformer, étiqueter, faire des marchés, défaire des marchés, courir partout... On s'est dit «oh, stop! Pourquoi tout cet argent? On dépense pas! C'est pas la peine!».

Associés à une fréquente pluriactivité, les systèmes de production et les modes de labellisation spécifiques à N\&P placent les agriculteurs des Cévennes à la marge de la profession agricole, comme le résume un couple installé depuis 22 ans en exploitant à titre principal: « Nous, on est à la marge d'une agriculture pour beaucoup de gens on fait pas de l'agriculture, on fait de la cueillette ».

\section{Conclusion}

L'invisibilité sociale peut être définie comme «un ensemble de processus, où interviennent des acteurs multiples, par lesquels un déni de reconnaissance des personnes aux divers niveaux de leur existence sociale peut affecter la profondeur, la durée et l'évolution de situations de pauvreté et d'exclusion» (Onpes, 2016 ; p. 39). La question de l'invisibilité est à mettre en vis-à-vis de celle de la reconnaissance et, à l'autre bout, celle de l'exclusion. Elle permet d'être objet de discours légitime. Il est clair que pour des producteurs promouvant d'autres modèles agricoles, l'enjeu de 
visibilité est fort puisque «la visibilité est le seul critère de l'action politique » (Tassin, 1991, cité par Onpes, 2016 ; p. 16).

Le texte montre une situation paradoxale pour les maraîchers N\&P rencontrés. D'une part une invisibilité géographique, académique et institutionnelle, mais d'autre part, une présence de plain-pied sur la scène des enjeux agroécologiques, de par leur résistance et leur opposition revendiquées à un certain type d'agriculture biologique. Leur posture rejoint celle du mouvement N\&P, auquel ils participent activement, et qui relève de l'idéal que poursuivaient les pionniers de l'agriculture biologique. Pour eux, l'agroécologie n'est pas circonscrite aux techniques agricoles mais relève d'un mouvement social (Wezel et al., 2009). Ils critiquent le règlement européen de la bio qui, selon eux, est bien en deçà des exigences écologiques et citoyennes revendiquées par ses pères fondateurs en $1991^{12}$, telles que le respect du vivant, le refus de l'industrialisation, ou le développement d'une agriculture familiale. Cette "vision très différente de l'agroécologie » de celle portée par le gouvernement, comme le souligne la fédération N\&P, peut mettre les pouvoirs publics en posture délicate. Car comment soutenir des modèles qui tournent le dos à l'agriculture productiviste, dans un système conçu pour ce type d'agriculture?

Paradoxalement, la mise à la marge de ces producteurs permet l'émergence de pratiques qui ont une vocation « rebelle » d'opposition au mot d'ordre des organisations majoritaires, montrant par ce biais qu'une autre façon de produire est possible.
Leur « invisibilité » ne donnerait-elle pas sens à leur mobilisation contre un modèle dominant (même s'il est biologique), celui-ci confirmant leurs différences en les rejetant? Car leur relative invisibilité politique ne les empêche pas de gagner du terrain parmi les professionnels en recherche de solutions alternatives: depuis 2008 , la fédération accueille chaque année en moyenne une cinquantaine d'adhérents professionnels sous mention, le nombre d'adhérents professionnels ayant quasiment doublé entre 2008 et $2016^{13}$ (N\&P, 2017, communication personnelle).

\section{Aurélie Javelle}

Ingénieure de recherche,

Montpellier SupAgro, UMR Innovation

Hélène Tallon

Consultante et chercheur associé UMR Innovation, Montpellier

\section{Bibliographie}

Jean-Marc Barbier, Frédéric Goulet, Moins de technique, plus de nature: pour une heuristique des pratiques d'écologisation de l'agriculture, Natures, Sciences, Sociétés, 21, pp. 200-210, 2013.

Maxime Catalogna, Analyse des logiques de gestion de la fertilité des sols en maraîchage biologique chez les agriculteurs cévenols, mémoire de fin d'étude d'ingénieur agronome, RESAD, Montpellier SupAgro, 2014.

Entente Départementale des Causses et Cévennes, Plan de gestion Causses et Cévennes, 2015-2021, UNESCO, 2014.

Nathalie Girard, Quels sont les nouveaux enjeux de gestion des connaissances? L'exemple de la transition écologique des systèmes agricoles, Revue internationale de psychosociologie et de gestion des comportements organisationnels, 49, 19 , pp. 51-78, 2014.

Paul de la Granville, Les pratiques agricoles de maraîchers cévenols dans leur 
contexte, Ébauche d'un champ de relations à l'environnement, M2 EDTS, AgroParisTech, 2016.

INRA, Vers des agricultures à hautes performances, Étude réalisée pour le Commissariat général à la stratégie et à la prospective, septembre 2013 (4 vol).

Ministère de l'Agriculture, de l'AgroAlimentaire et de la Forêt, Dix clefs pour comprendre l'agroécologie, MAAF, 2013.

Charte de la fédération Nature et Progrès, version avril 2013, en ligne: http:// www.natureetprogres.org/producteurs/ professionnels_nature_progres.php

Christian Nicourt, Etre agriculteur aujourd'hui. L'individualisation du travail des agriculteurs, ed. Quae, 2013

ONPES, L'invisibilité sociale: une responsabilité collective, Observatoire National de la Précarité et de l'Exclusion Sociale, 2016.

Diane Rodet, Des dispositifs de jugement pour et par les consommateurs? Les systèmes participatifs de garantie du commerce équitable, de l'agriculture biologique et des Amap, Revue Française de Socio-Économie, 2, 10, pp. 199-217, 2012.

Alexander Wezel, Stéphane Bellon, Thierry Doré, Charles A. Francis, Dominique Vallod, Christophe David, Agroecology as a science, a movement and a practice. A review, Agronomy for Sustainable Development, 29, 4, pp. 503-515, 2009.

1. http://www.natureetprogres.org/consulté le 14 mars 2014.

2. Cet avis a été entendu lors d'un travail de terrain avec des maraîchers N\&P, comme cela sera présenté plus loin.

3. Notamment la synthèse du volume 1 : Comment rendre l'agriculture biologique francaise plus productive et plus compétitive ? Voir un résumé de la polémique : http://institut.
inra.fr/Missions/Eclairer-les-decisions/ Etudes/Tous-les-dossiers/L-agriculturebiologique-en-debat

4. Les autres producteurs, étant en apiculture ou en élevage ont un rapport au sol moins fort, et n'ont donc pas été enquêtés.

5. Les bases consultées sont: Prodlnra, Cirad, Hal, Cemoa, Dumas, Francis, Pascal, Cairn, et google scholar, sur les termes «Nature et Progres» et «Nature \& Progres». Sur google scholar, 211 occurrences de «cultures en terrasse » ont été trouvées, une majorité en archéologie.

6. Règlement bio européen $834 / 2007$, mis en application en 2009, dénoncé dans un communiqué de presse de février 2016 commun à divers mouvements bio alternatifs. Le débat a été relancé en 2015 suite à des contrôles durcis de la DGCCRF (Direction Générale de la Concurrence de la Consommation et de la Répression des Fraudes).

7. Voir une analyse du SPG par Rodet, 2012.

8. Voir une brève histoire des débuts de l'association dans Nicourt, 2013, pp.199-201.

9. IFédération internationale du Mouvement de l'Agriculture Biologique.

10. Voir une analyse du SPG par Rodet, 2012.

11. Dotation Jeune Agriculteur, soumise à la présentation d'un diplôme agricole de niveau IV, et d'un plan de professionnalisation.

12. Voir communiqué de presse du 16 février 2016: http://www.natureetprogres.org/ actualite_agriculture_bio/actualite_agriculture_bio.html (consulté le 25/4/17).

13. Il est passé de 454 adhérents en 2008 à 824 en 2016. A noter que le nombre d'agriculteurs $A B$ a lui presque triplé dans la même période, passant de 11978 en 2007 à 32264 en 2016 (source dossier presse agence bio février 2017). 